\title{
Oculographic analysis of word reading in hemispatial neglect
}

\author{
M. Behrmann ${ }^{\text {a,* }}$, S.E. Black ${ }^{\text {b }}$, T.J. McKeeff ${ }^{\text {a }}$, J.J.S. Barton ${ }^{\mathrm{c}}$ \\ a Department of Psychology, Baker Hall, Carnegie Mellon University, Pittsburgh, PA 15213-3890, USA \\ ${ }^{\mathrm{b}}$ Cognitive Neurology Unit, Department of Medicine and the Ontario Heart and Stroke Foundation Centre for Stroke Recovery, \\ Sunnybrook and Women's College Health Sciences Centre, University of Toronto and Institute of Medical Sciences, \\ University of Toronto, 2075 Bayview Avenue, Toronto, Canada M4N 3M5 \\ ${ }^{\mathrm{c}}$ Department of Neurology, Beth Israel Hospital and Harvard Medical School, KS452 Beth Israel Deaconess Medical Center, \\ 330 Brookline Avenue, Boston, MA 02215, USA \\ Received 26 August 2002; accepted 9 September 2002
}

\begin{abstract}
Neglect dyslexia is a disorder in which individuals misread text appearing on the contralateral side of space following an acquired lesion, usually to the right parietal lobe. This disorder is generally attributed to an impairment in representing spatial information. To determine whether the spatial representations underlying reading differ from those mediating other forms of visual behavior, we investigated the cooccurrence of neglect dyslexia with that of neglect, which manifests on tasks such as line bisection or line cancellation. We also examined the correlation between neglect dyslexia, when present, and eye movements in order to characterize the neglect dyslexia disorder further. Whereas there is no clear relationship between the reading disorder and other symptoms of visuospatial neglect, suggesting segregated spatial representations, there is a direct correspondence between the oculomotor performance of patients with neglect dyslexia and their reading behavior. This latter result suggests that the reading deficit may well arise from the failure to register and perceive the contralesional information.
\end{abstract}

(C) 2002 Elsevier Science Inc. All rights reserved.

Keywords: Hemispatial neglect; Brain-damage; Reading; Neglect dyslexia; Eye movement; Oculomotor; Visuospatial neglect; Hemianopia

\section{Introduction}

Hemispatial neglect (or neglect for short) is a deficit in spatial representation, which, following brain-damage, afflicts humans and, to a lesser degree, nonhuman primates [1]. The central characteristic of this disorder is the failure to orient towards or report information that appears on the side of space opposite the lesion; for example, in humans, following a lesion to the right hemisphere, usually to parietal cortex, the individual may eat food from only the right side of the plate, dress only the right side of the body, and copy or draw only features on the ipsilesional right. Importantly, neglect occurs more often and with greater severity after right than left hemisphere lesions in humans, and so we refer to neglect as left-sided throughout this 2798.

* Corresponding author. Tel.: +1-412-268-2790; fax: +1-412-268-

E-mail address: behrmann+@cmu.edu (M. Behrmann). paper. The deficit can manifest in all sensory modalities, and can also give rise to slow and/or inaccurate contralateral manual or oculomotor responses. It is also the case that, when presented with printed material to read, the patients, all of whom are premorbidly literate, may also neglect the contralesional, left text. This specific disorder or 'neglect dyslexia' manifests in several ways: a patient may misread or omit text from the left page of a book entirely, or from the left side of the right page of text or even from the left portion of an individual word.

Although there are now several detailed characterizations of neglect dyslexia, the deficit appears to vary greatly across different individuals [2]. For example, some patients neglect the contralateral information of both isolated words and text, whereas others show neglect only in text reading or only in single word reading. Additionally, some patients neglect the left of words to a greater degree than the left of pronounceable nonwords (e.g., NABLE) whereas others remain unaffected by lexical status $[3,4]$. A further dimension of variation 
concerns the types of errors made. In general, the errors may take the form of omissions (e.g., TABLE $\rightarrow$ ABLE), substitutions (e.g., TABLE $\rightarrow$ CABLE), or additions (e.g., $\mathrm{TABLE} \rightarrow \mathrm{STABLE})$. In many patients, errors are predominantly of the substitution form [3,5-8] although this is by no means universal $[9,10]$, and some patients make mostly omission errors [11-13].

In addition to the heterogeneity within neglect dyslexia, it also remains unclear exactly how the reading deficit is related to the general neglect disorder, which is typically diagnosed on a series of standardized visual tasks. Among the standard tasks are (i) line bisection in which subjects are instructed to bisect a line and those with left neglect deviate rightwards in their bisection (neglecting a left-sided portion of the line) and (ii) line cancellation in which a display of many lines in different orientations is presented. In this latter task, subjects put a checkmark on each line to denote they have perceived it and those with left neglect omit to check lines on the contralesional left side. Although most patients with neglect dyslexia also exhibit visuospatial neglect on these standard tasks $[4,14]$, patients may show neglect dyslexia but not neglect on the other standard tasks [15-18] and vice versa $[10,19,20]$. The dissociation between neglect dyslexia and visuospatial neglect is also apparent after bilateral lesions in that some patients show left neglect dyslexia but right visuospatial neglect $[6,21,22]$. Understanding the apparent independence of visuospatial neglect and neglect dyslexia is critical as it suggests that the spatial representation of words may differ from the spatial representations used in other kinds of visual tasks. However, the heterogeneity across patients and the apparent dissociation between the behaviors may simply arise because different studies have used different procedures for characterizing the neglect dyslexia as well as the visuospatial neglect, and different clinical tests can yield differing results [23]. Additionally, because almost all studies describe single patients, who differ on so many dimensions, addressing the issue of unitary versus separate spatial representations has been difficult. The first goal of this study, then, is to examine the co-occurrence of visuospatial neglect and neglect dyslexia in a relatively large sample of individuals with a unilateral right hemisphere lesion to determine whether there is a segregation of spatial processes for reading and other forms of visual behavior.

The second goal of this study is to address an additional, more detailed aspect of neglect dyslexia. The particular issue concerns the relationship between the eye movement pattern of these patients and their reading deficit. It is not surprising, for example, that the patients fail to report the information from the contralateral side if they do not even saccade to that side. It has been suggested, however, that this is not the case and that neglect patients fail to acknowledge the presence of contralesional stimuli even after fixating them for a prolonged period of time. If this latter situation were so, it would be consistent with an explanation of neglect (and neglect dyslexia) as a higher-order disorder of spatial awareness that cannot be attributed to a more fundamental deficit in which the stimuli are simply not registered or encoded adequately. To the extent that this oculomotor-behavior relationship has been studied, the data are contradictory: many studies have demonstrated an association between the two, such that patients make few contralesional saccades and also do not report the contralateral information $[24,25]$. A few recent studies, however, have shown that even when the patients fixate the material, they still fail to report it [26-28]. To explore this issue further, we initially examine the duration and number of fixations made to the left and right by neglect patients with and without neglect dyslexia, compared with two other groups of control subjects. We then evaluate in the neglect dyslexia patients, on a word-by-word basis, the association between the behavioral performance and the eye movements for those words read correctly compared with those read incorrectly.

\section{Method}

\subsection{Subjects}

Subjects were right-handed, native English speakers with visual acuity of at least 20/40 with correction, and all consented to participate.

The neglect group consisted of nine patients (five male, four female) with left neglect following a CT- or MRIdocumented unilateral cerebral lesion. Neglect was diagnosed on the basis of a standardized battery of examinations (for details, see Refs. [29,30]) where a score of 6 or greater indicates neglect and higher scores denote increased severity. The mean age and mean education level of the patients were 62 (S.D. 5.9) years and 11.3 (S.D. 5.1) years, respectively. All had a visual field defect to some degree, measured using Humphrey automated threshold perimetry (30-2 program). Demographic details, lesion site and volume, visual field status, and neglect scores are shown in Table 1, and templates depicting the lesions are illustrated in Fig. 1 (unfortunately, no scan was available for patient 10, CP). All of the patients, with the exception of $\mathrm{CP}$, have participated in previous eye tracking studies [24,31], and the reader is referred to those papers for further details. Note that, in Table 1, the patient numbers have been labeled so as to be consistent with Ref. [24] to allow the reader to compare subject performance across the two papers. Patient AG, who participated in that study, was not included here as he was not a native English speaker.

The hemianopic group consisted of four patients (one female, three male), none of whom had neglect. The mean age of the group was 53.8 (S.D. 17.9) years. None of these subjects had cataracts, retinopathy or glaucoma, and all had visual field defects (Humphrey automated threshold perimetry 30-2 program). The demographics and scores on 
Table 1

Demographic and lesion data for neglect and hemianopic patients

\begin{tabular}{|c|c|c|c|c|c|c|c|}
\hline Patient & Sex & Age & Years ed. & Spatial neg. score & Time test. ${ }^{\mathrm{a}}$ & Lesion $^{b}$ & Volume \\
\hline \multicolumn{8}{|l|}{ Neglect } \\
\hline 2. AR & M & 62 & 11 & 16 & 3 & $\mathrm{O}$ & 46.6 \\
\hline 3. DD & $\mathrm{F}$ & 61 & 13 & 13 & 24 & $\mathrm{O}$ & 85.2 \\
\hline 4. ET & $\mathrm{F}$ & 67 & 14 & 78 & 9 & $\mathrm{~F}, \mathrm{P}, \mathrm{T}, \mathrm{BG}, \mathrm{Th}, \mathrm{IC}$ & 129.4 \\
\hline 5. HL & $\mathrm{F}$ & 76 & 15 & 16 & 10 & $\mathrm{P}, \mathrm{T}, \mathrm{IC}, \mathrm{BG}$ & 31.4 \\
\hline 6. HI & M & 74 & 17 & 94 & 23 & $\mathrm{~F}, \mathrm{~T}, \mathrm{P}$ & 15 \\
\hline 7. JR & $\mathrm{F}$ & 73 & 11 & 37 & 4 & $\mathrm{~F}, \mathrm{P}, \mathrm{T}, \mathrm{IC}, \mathrm{BG}$ & 61.2 \\
\hline 8. FR & M & 78 & 12 & 97 & 7 & $\mathrm{~T}, \mathrm{P}, \mathrm{O}$ & 36 \\
\hline 9. $\mathrm{OH}$ & $\mathrm{F}$ & 74 & 9 & 88 & 11 & $\mathrm{~T}, \mathrm{O}, \mathrm{BG}, \mathrm{Th}, \mathrm{IC}$ & 39.6 \\
\hline 10. CP & M & 57 & $?$ & 100 & 0.25 & $\mathrm{P}, \mathrm{Th}$ & $?$ \\
\hline \multicolumn{8}{|c|}{ Hemianopic } \\
\hline 1. SS & $\mathrm{F}$ & 28 & 13 & 0 & 14 & $\mathrm{O}, \mathrm{T}$ & 103 \\
\hline 2. WH & M & 55 & $?$ & 0 & 11 & $\mathrm{O}, \mathrm{T}, \mathrm{P}$ & 86.5 \\
\hline 3. DL & M & 66 & 13 & 2 & 13 & $\mathrm{P}, \mathrm{T}, \mathrm{BG}$ & 25.8 \\
\hline 4. $\mathrm{PW}$ & M & 66 & 18 & 1 & 22 & $\mathrm{O}, \mathrm{Th}$ & 111.9 \\
\hline
\end{tabular}

a Time of testing post-onset in months.

${ }^{\mathrm{b}} \mathrm{F}=$ frontal, $\mathrm{P}=$ parietal, $\mathrm{T}=$ temporal, $\mathrm{O}=$ occipital, $\mathrm{IC}=$ internal capsule, $\mathrm{BG}=$ basal ganglia, $\mathrm{Th}=$ thalamus.

neglect testing are shown in Table 1. The control group consisted of nine subjects (four male, five female) with no history of neurological disease and no neglect. Their mean age (59.2; S.D. 3.4) and years of education (13.1; S.D. 2.9) were not significantly different from those of the neglect patients (both $F<1$ ).

\subsection{Apparatus}

Subjects were seated in a chair in a dimly illuminated room with the head supported in an occipital rest. Eye position and movement were measured using the magnetic search coil technique with 6-ft field coils (CNC Engineering, Seattle, WA). System bandwidth was $0-400 \mathrm{~Hz}$. Subjects wore a scleral contact annulus in one eye while they viewed the target display. The system had a resolution, after analogue to digital conversion, of about $1 \mathrm{~min}$. At the beginning of the session, the coil was placed in the right eye following a drop of topical anesthetic, and it remained in place for about $30 \mathrm{~min}$. A screen on which the stimuli were presented was located $1.14 \mathrm{~m}$ from the subject and viewing was binocular.

The signal from the eyetracker was sampled every $5 \mathrm{~ms}$ (i.e., 200 samples/s) by computer. The analytic program identified the start and end of saccades. Fixations were defined as the interval of stable horizontal and vertical eye position between the end of one saccade and the start of the following saccade: the output of the algorithm was a series of horizontal $(x)$ and vertical $(y)$ coordinates of each fixation period and its corresponding fixation duration $(z)$. The analogue signals were digitized and stored on a hard disk off-line for later analysis, using an interactive program on a PDP11/73 computer. Eye position was also recorded simultaneously on a rectilinear ink-jet polygraph (ElemaSchönander, Stockholm).

\subsection{Procedure}

\subsubsection{Calibration}

Before collecting the data on the reading task, the signals from the coil were calibrated by having the subject fixate spots of light located at various places on the screen. A red spot of light, subtending $1^{\circ}$ of visual angle, was backprojected onto the center of the screen and the zero point $(0,0)$ calibration was verified. The subject was then instructed to look at a black dot $\left(0.5^{\circ}\right.$ of visual angle), which appeared at each of the four corners of a large screen, placed in front of the subjects (each corner was located at $\pm 22.5^{\circ}$ horizontal and $\pm 18^{\circ}$ vertical). Fixation of these five positions (zero and the corners) was repeated three times to establish the perimeter and center of the board, and then the experiment began. The zero point calibration was repeated again after the experiment to ensure that no shifts in coordinates had taken place.

\subsubsection{Word reading task}

Subjects read two sets of 15 words consisting of 5 words each of 4, 5 and 6 letters. All words were chosen so that omission of the left letter would give rise to another English word. This often induces more severe neglect [5], and longer words tend to give rise to greater neglect [5]. The words were laid out in five columns, one spanning the midline and two to the right and left, respectively (see Fig. $2 \mathrm{a}$ for example). Each column contained one word of each word length. The words were displayed on a large board, subtending $45^{\circ}\left( \pm 22.5^{\circ}\right)$ and $36^{\circ}\left( \pm 18^{\circ}\right)$ of visual angle horizontally and vertically, respectively. This arrangement has proven useful for measuring performance of neglect patients [3,24,32], and recent evidence has suggested that the larger the spatial extent of the stimulus array, the more severe the neglect deficit [33]. The letters were printed in 


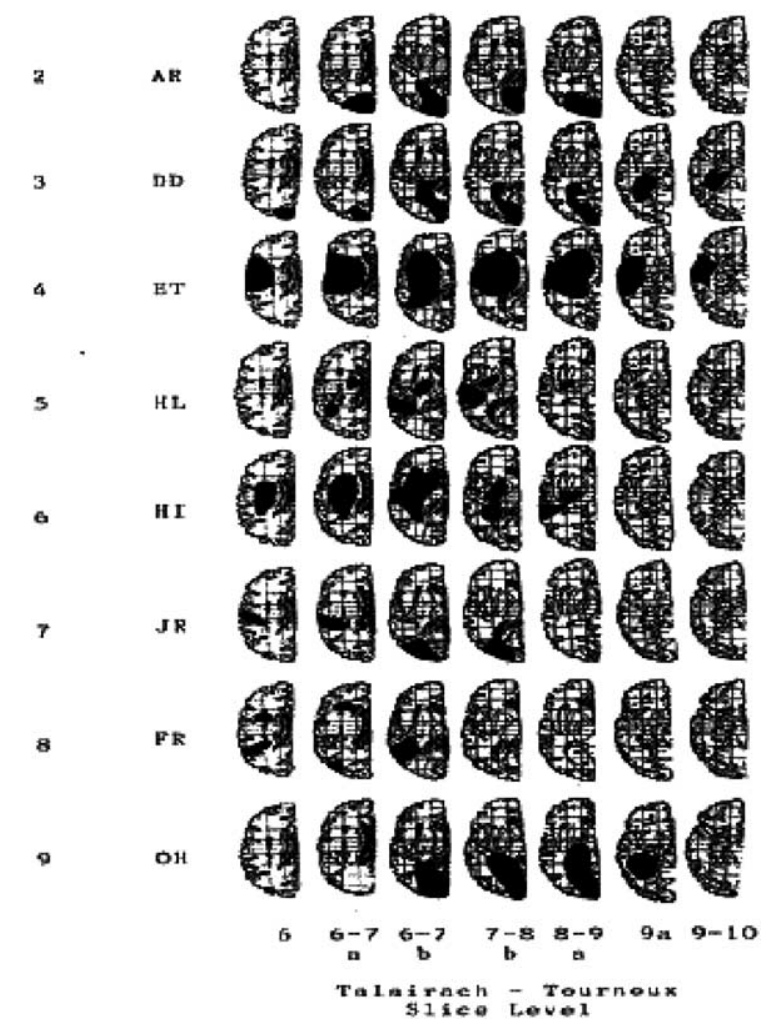

Fig. 1. Depiction of lesion sites for the neglect patients on standardized templates, adapted from Ref. [35].

black ink in bold upper case Geneva font on a white background, and each letter subtended $1^{\circ}$ of visual angle, well within the resolution of the eye tracker. Subjects were instructed to read each word aloud and to notify the examiner when they were finished. The order of the two word sets was counterbalanced across participants. A tape recording of the verbal output of the subject was made at the same time as the eye tracking, and reading errors were noted.

\section{Results}

\subsection{Neglect dyslexia and visuospatial neglect}

To examine the association between neglect dyslexia and visuospatial neglect, we first classified subjects according to the presence or absence of neglect dyslexia. To do this, based on the verbal output of the patients, we counted the number of words omitted entirely and the number of individual words misread and these are shown in Table 2. Only those words containing an error to the left of the 'neglect point' [3] were counted as a neglect error. A left neglect error was defined as a response in which all letters to the left of a certain location in the word were read incorrectly without exception and all letters to the right were read correctly; for example, SEND $\rightarrow$ TEND (neglect error, with neglect point between $\mathrm{S}$ and $\mathrm{E}$ ) versus $\mathrm{SEND} \rightarrow$ SAND (not a neglect error). Of note, the hemianopic patients and normal control subjects read all the items correctly. In contrast, as is evident from this table, there are four profiles of behavior in the neglect subgroup: (i) those without neglect dyslexia (Patients 4, 6, 7 and 9); (ii) those who misread entire words and also make partial errors on words (Patients 2, 8 and 10); (iii) those who neglect entire words but not partial words (Patient 5); and (iv) those who make partial errors on words but do not neglect entire words (Patient 3). Patients in subgroups (ii)-(iv) are considered to have neglect dyslexia.

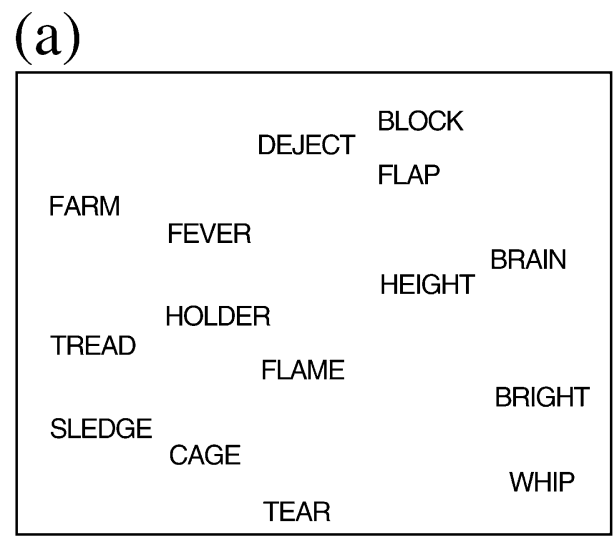

(b)

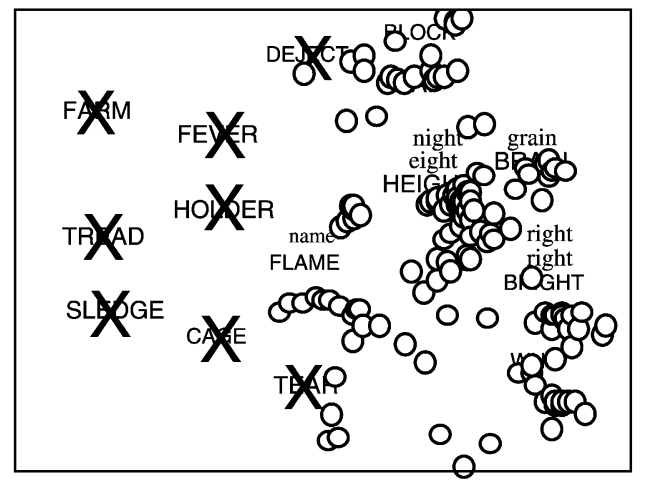

(c)

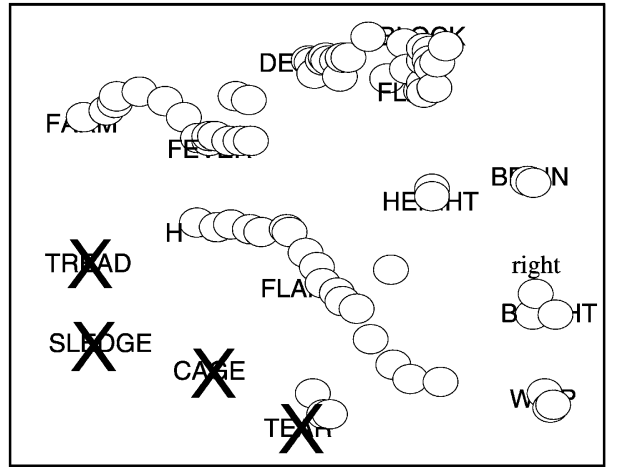

Fig. 2. Layout of experimental set-up for reading and eye movement data collection. (a) Example of stimulus board for reading. (b, c) The circles reflect the locations of fixations. A ' $\mathrm{X}$ ' indicates that the word was omitted and the lower case word is the reading error made by the patient. (b) The performance of Patient 2 and (c) the performance of Patient 8 . 
Table 2

Breakdown of reading errors for whole and partial words in patients with neglect

\begin{tabular}{|c|c|c|c|c|}
\hline Patient & $\begin{array}{l}\text { Spatial } \\
\text { neglect } \\
\text { score }\end{array}$ & $\begin{array}{l}\text { Whole word } \\
\text { correct } \\
(n=30)\end{array}$ & $\begin{array}{l}\text { Whole words } \\
\text { omitted }\end{array}$ & $\begin{array}{l}\text { Partial words neglected } \\
\text { omissions substitutions }\end{array}$ \\
\hline 2. AR & 16 & 12 & 12 & 2 \\
\hline 3. DD & 13 & 23 & 0 & 5 \\
\hline 4. ET & 78 & 30 & 0 & 0 \\
\hline 5. HL & 16 & 25 & 5 & 0 \\
\hline 6. HI & 94 & 30 & 0 & 0 \\
\hline 7. JR & 37 & 30 & 0 & 0 \\
\hline 8. FR & 97 & 9 & 14 & 6 \\
\hline 9. $\mathrm{OH}$ & 88 & 30 & 0 & 0 \\
\hline 10. CP & 100 & 4 & 26 & 1 \\
\hline
\end{tabular}

Of note, there is no obvious association between the presence and severity of spatial neglect and the reading profile, as determined by the spatial neglect score and the reading errors. Indeed, three of the four patients with no reading errors have among the highest spatial neglect scores. Additionally, one of the two patients who neglected the left of words and the left of text has very mild spatial neglect (Patient 2: neglect score 16; Fig. 2b) while the other has very severe spatial neglect (Patient 8: neglect score 97; Fig. $2 \mathrm{c})$. The lack of obvious correspondence between the neglect dyslexia and neglect score suggests that different representations might well underlie word reading versus other visual tasks. This finding is consistent with previous studies on the dissociation of reading and other visual behaviors.

\subsection{Neglect dyslexia and eye movements}

To determine whether patients fixate words they fail to read or the reverse, we examined the correlation between the behavior and the eye movements of the patients. We did this in two ways: first, we documented the number of fixations and durations on the right versus left of the displays for the two neglect subgroups, those with and without neglect dyslexia, in comparison to the control and hemianopic groups. We predicted that only the neglect dyslexic patients would show a reduction in the number of fixations and duration on the contralesional side. Second, for the neglect patients, we ascertained on an item-by-item basis, the correlation between the words fixated and their reported accuracy. For this analysis, we defined a fixation as being on the word or letter if it fell within $0.5^{\circ}$ of the stimulus. Fig. 2 shows the layout of one of the two sets of words with the whole and partial word errors made by neglect dyslexic Patients (b) 2 and (c) 8 . The circles denote the locations of the fixations in relation to the words.

An analysis of variance with group (controls, hemianopics, those with neglect dyslexia, those without neglect dyslexia) as a between-subjects factor, and word length and position (left, right) as within-subject factors, revealed a significant interaction between these three variables, $F(6$, $34)=2.9, P=.02$. The same was true with duration as the dependent measure, $F(6,34)=3.1, P<.02$. As is evident from Fig. 3, the normal control subjects and those with hemianopia show no difference in the number of fixations or duration thereof for the left versus right of the display. The same is true of those without neglect dyslexia although there is a trend, albeit nonsignificant, to make more left-sided fixations and fixations of longer duration, perhaps as a form of compensation for the neglect. In contrast, patients with neglect dyslexia make very few fixations to the left and if they do, their fixations are brief in duration. Of note, while the three other groups show a slight increment in the number of fixations and duration over word length, the neglect dyslexia group is not sensitive to this factor. In addition to the differences on the contralesional side, the neglect

(a)

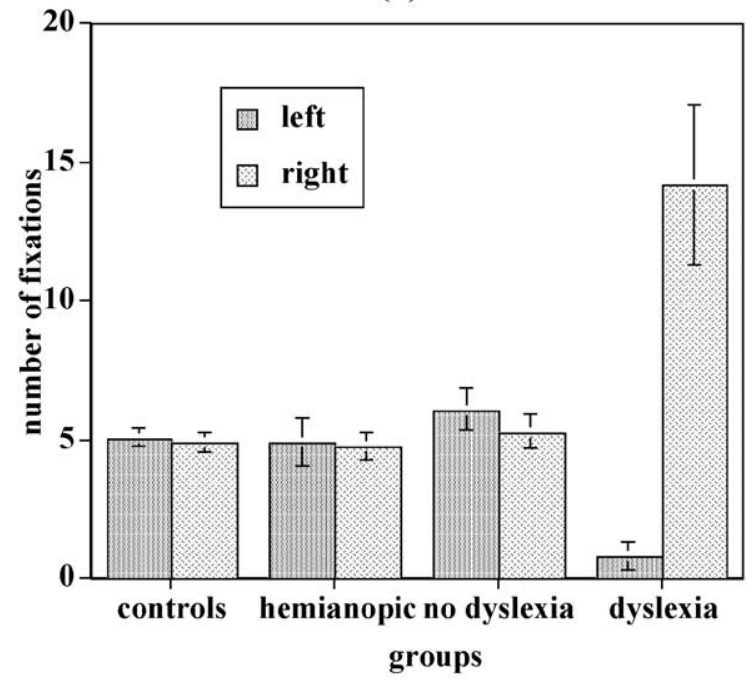

(b)

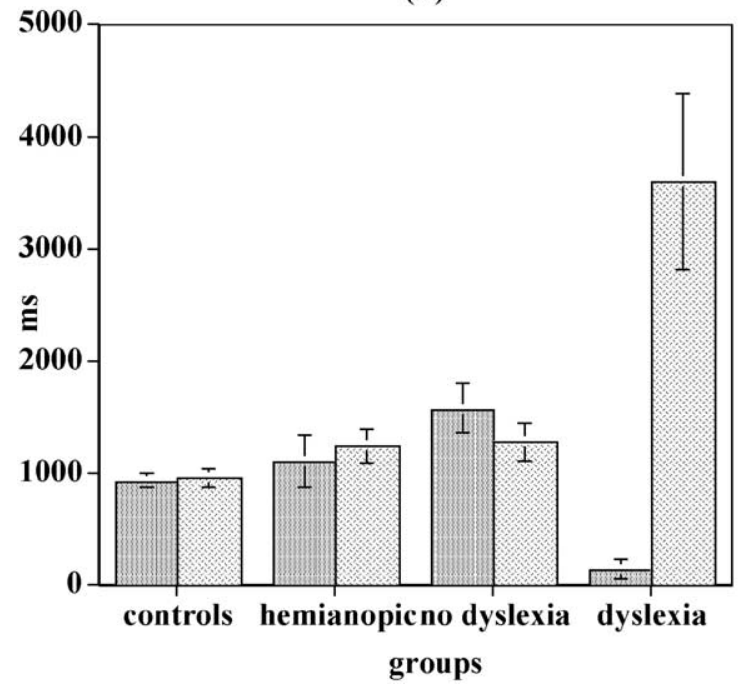

Fig. 3. Mean (a) number of fixations and (b) duration of fixations for the control subjects, hemianopic patients, brain-damaged patients without neglect dyslexia and brain-damaged patients with neglect dyslexia. 
Table 3

Association between whole words read accurately or not and fixated or not

\begin{tabular}{lll}
\hline Whole words & Eye movements & \\
\cline { 2 - 3 } & Fixated & Not fixated \\
\hline Read & 71 & 11 \\
Not read & 8 & 60
\end{tabular}

dyslexia individuals make many more fixations and fixations of longer duration to the ipsilesional right than any of the other groups. The hyperattention to the ipsilateral side and paucity of representation of the contralateral side are thought of as two sides of the same coin in neglect [24]. Both are well-known features of neglect performance and manifest here in the domain of reading. Note that this leftright discrepancy may even be underestimated in the current study as we have also included in the neglect dyslexia group those patients who do not err on whole words but only on partial words; these individuals do make some saccades to the left, thereby boosting the number of contralesional saccades.

The second measure is more fine-grained. Table 3 lays out the contingencies between words fixated/not fixated and $\mathrm{read} /$ not read correctly. As is evident, there is a very strong relationship between fixation and correct report $\left(\chi_{1}^{2}=80.5\right.$, $P<.0001)$, suggesting that those items that are fixated are read correctly whereas those that are not fixated are not read correctly. This analysis takes into account whole words that are not read correctly as well as words that are only read partially correctly.

\section{Discussion}

The primary focus of this study is on neglect dyslexia, a spatial deficit that affects reading and arises following a unilateral hemispheric lesion in premorbidly literate individuals. Two major issues were addressed in this work. The first is the association between neglect dyslexia and neglect on other visual tasks, with the prediction that when two behaviors are strongly associated, and break down in tandem, they are likely to be mediated by the same underlying spatial representation. The second issue addresses the association between neglect dyslexia and eye movements. Whereas some studies have found that individuals fixate items and still fail to report them correctly, other studies have found a direct correspondence between behavioral report and oculomotor behavior. If patients do fixate items but do not report them, this would suggest that neglect does not arise from a deficit in encoding the visual stimulus; instead, it might be attributable to a higher-order problem in awareness or in the selection of the encoded information. In contrast, the failure to fixate stimuli on the contralateral side and the corresponding failure to report them suggests that neglect dyslexia may be a direct consequence of the failure to register and to perceive the material.
We have found a clear dissociation between the presence of neglect dyslexia and visuospatial neglect. Although this dissociation has been reported previously, it has not been systematically explored. To our knowledge, only one other study to date has reported the co-occurrence of neglect dyslexia and visuospatial neglect in a large sample of patients. Consistent with our results, a previous study showed the presence of neglect dyslexia in only 9 out of 34 patients [13]. These findings support the independence of representations for word reading and other forms of visual behavior.

We have, on the other hand, obtained a strong association between fixation and behavioral report, as reflected in the strong correlation between them. These data suggest that the contralateral information is not obviously being registered and that the failure to report the words might be a direct consequence of the failure to encode them in the first place. Indeed, previous studies that we have undertaken demonstrate that patients with neglect make few saccades to the contralateral side, especially if there is a concurrent ipsilateral stimulus [34], as in the present paradigm. It may well be the case, therefore, that the observed neglect dyslexia is a direct consequence of this eye movement pattern. The current findings, then, challenge views that argue for a deficit in spatial awareness or in the selection of information that has been perceived and encoded. Instead, a simpler explanation might hold in which the neglect dyslexia is a direct consequence of a deficit in extracting perceptual information from the left side of space. Taken together, our data serve to clarify some of the outstanding issues regarding neglect dyslexia, and to illustrate the profitability of a neuropsychological approach for understanding how visual information is processed and represented.

\section{Acknowledgements}

This research was supported by a NIMH award to MB (MH54216), by the Canadian Institute for Health Research and by the Heart and Stroke Foundation of Ontario. The authors thank Phat Nguyen, Jane Collins, Shemira Murji and Patricia Ebert for help with data collection and analysis, and Dr. J. Sharpe for his input and support.

\section{References}

[1] Duhamel JR, Wardak C. Is there a monkey analogue of human neglect? In: Karnath HO, Milner AD, Vallar G, editors. Cognitive and neural bases of spatial neglect. Oxford (UK): Oxford Univ Press; 2003.

[2] Haywood M, Coltheart M. Neglect dyslexia and the early stages of visual word recognition. Neurocase 2000;6:33-43.

[3] Ellis AW, Flude B, Young AW. Neglect dyslexia and the early visual processing of letters in words and nonwords. Cogn Neuropsychol 1987;4:439-64. 
[4] Subbiah I, Caramazza A. Stimulus-centered neglect in reading and object recognition. Neurocase 2000;6:13-30.

[5] Behrmann M, Moscovitch M, Black SE, Mozer M. Perceptual and conceptual factors in neglect dyslexia: two contrasting case studies. Brain 1990;113:1163-83.

[6] Cubelli R, Nichelli P, Bonito V, Tanti AD, Inzhagi M. Different patterns of dissociation in unilateral spatial neglect. Brain Cogn 1991; 15:139-59.

[7] Kinsbourne M, Warrington EK. A variety of reading disability associated with right hemisphere lesions. J Neurol Neurosurg Psychiatry 1962;25:339-44.

[8] Riddoch MJ, Humphreys GW, Cleton P, Fery P. Interaction of attentional and lexical factors in neglect dyslexia. Cogn Neuropsychol 1990;7:479-517.

[9] Caramazza A, Hillis AE. Levels of representation, co-ordinate frames and unilateral neglect. Cogn Neuropsychol 1990;13:391-446.

[10] Warrington EK. Right neglect dyslexia: a single case study. Cogn Neuropsychol 1991;8:193-212.

[11] Cubelli R, Simoncini L. Dissociation between word reading and word copying in a patient with left visual neglect. Cortex 1997;33:177-85.

[12] Vallar G, Guariglia C, Nico D, Tabossi P. Left neglect dyslexia and the processing of neglected information. J Clin Exp Neuropsychol 1996; $18: 1-14$.

[13] Làdavas E, Umilta C, Mapelli D. Lexical and semantic processing in the absence of word reading: evidence from neglect dyslexia. Neuropsychologia 1997;35:1075-85.

[14] Miceli G, Capasso R. Word-centred neglect dyslexia: evidence from a new case. Neurocase 2001;7:221-36.

[15] Haywood M, Coltheart M. Neglect dyslexia with a stimulus-centred deficit and without visuospatial neglect. Cogn Neuropsychol 2001; 18:577-615.

[16] Cantoni C, Piccirilli M. Dissociation in the recovery from neglect dyslexia and visuospatial unilateral neglect. Ital J Neurol Sci 1997; $18: 41-3$.

[17] Kartsounis LD, Warrington EK. Unilateral visual neglect overcome by cues implicit in stimulus arrays. J Neurol Neurosurg Psychiatry 1989; 52:1253-9.

[18] Bisiach E, Vallar G, Perani D, Papagno C, Berti A. Unawareness of disease following lesions of the right hemisphere: anosagnosia for hemiplegia and anosagnosia for hemianopia. Neuropsychologia 1986;24:471-82.

[19] Katz RB, Sevush S. Positional dyslexia. Brain Lang 1989;37:266-89.
[20] Patterson KE, Wilson B. A rose is a nose: a deficit in initial letter identification. Cogn Neuropsychol 1990;13:447-78.

[21] Costello AD, Warrington EK. The dissociation of visual neglect and neglect dyslexia. J Neurol Neurosurg Psychiatry 1987;50:1110-6.

[22] Riddoch MJ, Humphreys GW, Luckhurst L, Burroughs E, Bateman A. Paradoxical neglect: spatial representations, hemisphere-specific activation and spatial cueing. Cogn Neuropsychol 1995;12:569-604.

[23] Halligan PW, Marshall JC. Toward a principled explanation of unilateral neglect. Cogn Neuropsychol 1994;11:167-206.

[24] Behrmann M, Barton JJS, Watt S, Black SE. Impaired visual search in patients with unilateral neglect: an oculographic analysis. Neuropsychologia 1997;35:1445-58.

[25] Karnath HO, Niemeier M. Task-dependent differences in the exploratory behaviour of patients with spatial neglect. Neuropsychologia 2002;40:1577-85.

[26] Làdavas E, Zeloni G, Zaccara G, Gangemi P. Eye movements and orienting of attention in patients with visual neglect. J Cogn Neurosci 1997;9:67-74.

[27] Walker R, Findlay JM, Young AW, Lincoln NB. Saccadic eye movements in object-based neglect. Cogn Neuropsychol 1996;13:569-615.

[28] di Pellegrino G, Ladavas E, Galletti C. Lexical processes and eye movements in neglect dyslexia. Behav Neurol 2001/2002;13:61-74.

[29] Black SE, Vu B, Martin D, Szalai JP. Evaluation of a bedside battery for hemispatial neglect in acute stroke. J Clin Exp Neuropsychol [abstract] 1990;12:102.

[30] Black SE, Ebert P, Leibovitch F, Szalai J, Blair N, Bondar J. Recovery in hemispatial neglect. Neurology 1994;45:A178.

[31] Barton JJS, Behrmann M, Black SE. Ocular search during line bisection: the effects of hemineglect and hemianopia. Brain 1998;121: $1117-31$.

[32] Geng JJ, Behrmann M. Implicit cueing by statistical contingencies affects visual search in normal participants and patients with hemispatial neglect. Psychol Sci 2002;13(6):520-5.

[33] Eglin M, Robertson LC, Knight RT, Brugger P. Search deficits in neglect patients are dependent on size of the visual scene. Neuropsychology 1994;8:451-63.

[34] Behrmann M, Ghiselli-Crippa T, Sweeney J, Dimatteo I, Kass R. Mechanisms underlying spatial representation revealed through studies of hemispatial neglect. J Cogn Neurosci 2002;14:272-90.

[35] Talairach J, Tournoux P. Co-planar stereotaxic atlas of the human brain. New York: George Thieme Verlag; 1988. 